PREPARED FOR THE U.S. DEPARTMENT OF ENERGY, UNDER CONTRACT DE-AC02-76CH03073

PPPL-3981

PPPL-3981

UC-70

\author{
Extraction of Poloidal Velocity \\ from Charge Exchange Recombination \\ Spectroscopy Measurements
}

by

W.M. Solomon, K.H. Burrell, P. Gohil, R.J. Groebner, and L.R. Baylor

July 2004

NM|

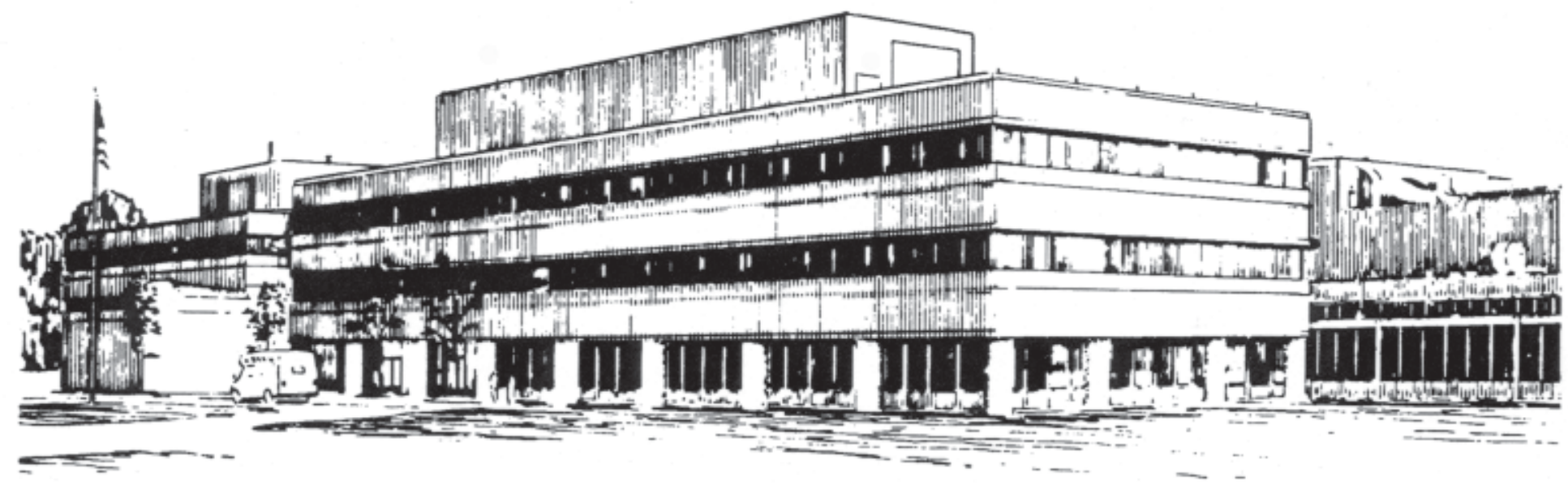

PRINCETON PLASMA PHYSICS LABORATORY PRINCETON UNIVERSITY, PRINCETON, NEW JERSEY 


\section{PPPL Reports Disclaimer}

This report was prepared as an account of work sponsored by an agency of the United States Government. Neither the United States Government nor any agency thereof, nor any of their employees, makes any warranty, express or implied, or assumes any legal liability or responsibility for the accuracy, completeness, or usefulness of any information, apparatus, product, or process disclosed, or represents that its use would not infringe privately owned rights. Reference herein to any specific commercial product, process, or service by trade name, trademark, manufacturer, or otherwise, does not necessarily constitute or imply its endorsement, recommendation, or favoring by the United States Government or any agency thereof. The views and opinions of authors expressed herein do not necessarily state or reflect those of the United States Government or any agency thereof.

\section{Availability}

This report is posted on the U.S. Department of Energy's Princeton Plasma Physics Laboratory Publications and Reports web site in Fiscal Year 2004. The home page for PPPL Reports and Publications is: http://www.pppl.gov/pub_report/

DOE and DOE Contractors can obtain copies of this report from:

U.S. Department of Energy

Office of Scientific and Technical Information

DOE Technical Information Services (DTIS)

P.O. Box 62

Oak Ridge, TN 37831

Telephone: (865) 576-8401

Fax: (865) 576-5728

Email: reports@adonis.osti.gov

This report is available to the general public from:

National Technical Information Service

U.S. Department of Commerce

5285 Port Royal Road

Springfield, VA 22161

Telephone: $1-800-553-6847$ or

(703) $605-6000$

Fax: (703) 321-8547

Internet: http://www.ntis.gov/ordering.htm 


\title{
Extraction of poloidal velocity from charge exchange recombination spectroscopy measurements
}

\author{
W. M. Solomon* \\ Princeton Plasma Physics Laboratory, \\ Princeton University, Princeton, NJ 08543 \\ K. H. Burrell, P. Gohil, and R. J. Groebner \\ General Atomics, P.O. Box 85608, San Diego, California 92186-5608 \\ L. R. Baylor \\ Oak Ridge National Laboratory, Oak Ridge, Tennessee
}

(Dated: July 7, 2004)

\begin{abstract}
A novel approach has been implemented on DIII-D to allow the correct determination of the plasma poloidal velocity from charge exchange spectroscopy measurements. Unlike usual techniques, the need for detailed atomic physics calculations to properly interpret the results is alleviated. Instead, the needed atomic physics corrections are self-consistently determined directly from the measurements, by making use of specially chosen viewing chords. Modeling results are presented that were used to determine a set of views capable of measuring the correction terms. We present the analysis of a quiescent H-mode discharge, illustrating that significant modifications to the velocity profiles are required in these high ion temperature conditions. We also present preliminary measurements providing the first direct comparison of the standard cross-section correction to the atomic physics calculations.
\end{abstract}

*Electronic address: wsolomon@pppl.gov 


\section{INTRODUCTION}

Rotation plays an important role in plasma confinement and stability, yet it is still poorly investigated. To further our understanding and predictive capability, we are interested in making a detailed comparison of the neoclassical theory of rotation with poloidal rotation measurements. Due to the generally small poloidal rotations observed on DIII-D, one must make very accurate measurements in order to make a good test against theory. The technique of charge exchange recombination spectroscopy (CER) [1] for rotation measurements is well-known. However, there are various atomic physics corrections that are particularly significant for poloidal velocity measurements from CER [2]. Since these effects can be of the same order as the poloidal velocity, it is essential that the atomic physics is accurately handled. In previous work [2], the atomic physics quantities were calculated based on extensive and complicated modeling. Even so, there remains uncertainty in the various cross-sections required for such modeling. Furthermore, despite the modeling efforts, data from TFTR could not be fully reconciled. Hence, an alternate technique, based on direct measurement of the corrections, is preferable. This possibility has been realized on DIII-D using a specialized set of viewing chords.

In Sec. II, the theory for the apparent velocity as measured by the CER system is presented, taking into account the atomic physics corrections. Section III illustrates conceptually how one can make a set of measurements to separate the true poloidal rotation from the corrections. Modeling supporting the choice of views required to self-consistently determine the poloidal rotation and atomic physics corrections is presented in Sec. IV. Finally, some proof-of-principle results are shown in Sec. V, as well as a preliminary verification of the energy dependence of the charge exchange cross-sectional area.

\section{THEORY}

The emission from the charge exchange process is proportional to the product of neutral beam density, the impurity ion density, and the effective emission rate, $E \propto n_{b} n Q$. The emission rate is given by $Q\left(\left|\vec{v}-\vec{v}_{b}\right|\right)=\sigma^{C X}\left(\left|\vec{v}-\vec{v}_{b}\right|\right)\left|\vec{v}-\vec{v}_{b}\right|$, where $\sigma^{C X}$ is the charge

exchange cross-sectional area, $\vec{v}$ is the velocity of the impurity ion, and $\vec{v}_{b}$ is the neutral beam velocity. Several atomic physics effects come into play when measuring the charge exchange 
emission in hot plasmas, due to the energy dependence of the charge exchange cross-section, $\sigma^{C X}=\sigma^{C X}\left(\left|\vec{v}-\vec{v}_{b}\right|\right)$. The effective emission rate for the $D^{0}+C^{6+} \rightarrow D^{+}+C^{5+}(n=8 \rightarrow 7)$ transition is shown in Fig. 1 [3]. Typical neutral beam energies $(<100 \mathrm{keV})$ put us on the left side of the maximum. Hence, an ion moving towards the neutral beam, and therefore with a slightly higher collision velocity, is more likely to undergo charge exchange than an ion moving away from the beam. This results in one side of the emission profile being artificially enhanced, which looks like an apparent line shift normally associated with Doppler motion. The effect scales with the ion temperature $T_{i}$ and is only present when a viewing chord has a component parallel to the neutral beam.

If this were the full story, then poloidal velocity measurements, which are usually made with vertical views perpendicular to the neutral beam, would not suffer any atomic physics corrections. However, an additional complication arises from the fact that, during the finite lifetime $\tau$ of the excited energy state, the ions travel $\omega_{c} \tau$ (where $\omega_{c}$ is the ion cyclotron frequency) around their gyro-orbit . In this way, an apparent radial velocity can be transformed into an apparent poloidal velocity [2]. The effect in TFTR, with high $T_{i} \sim 20-30 \mathrm{keV}$ and magnetic field $B \sim 2-5 \mathrm{~T}$, was very significant, with apparent poloidal velocities up to $40 \mathrm{~km} / \mathrm{s}$.

We derive the effect of the energy-dependent cross-section correction coupled with the gyro-motion of the ions on the measured velocity. The distribution function $f_{n}(\vec{v})$ of an ion with charge $Z_{n}$, which is created from an ion of charge $Z_{n+1}$ through charge exchange with a beam neutral, is described by the Boltzmann equation

$$
\frac{\partial f_{n}}{\partial t}+\frac{Z_{n} e}{m} \vec{v} \times \vec{B} \cdot \vec{\nabla}_{v} f_{n}=n_{b} Q\left(\left|\vec{v}-\vec{v}_{b}\right|\right) f_{n+1}(\vec{v}-\vec{V})-\frac{f_{n}}{\tau},
$$

where $m$ is the ion mass and $\vec{V}$ is the average ion velocity. This form neglects cascading from more than one energy level, although this can be handled in part by substituting an "effective" lifetime for $\tau$. With the distribution function, we can compute the apparent velocity

$$
\vec{v}_{a p p}^{l o c a l}=\frac{\int d^{3} v \vec{v} f_{n}}{\int d^{3} v f_{n}} .
$$

Note that this form implies a local measurement of the distribution function to give a local apparent velocity. Most CER measurements, however, are intrinsically line-of-sight (LOS). Nonetheless, we will develop the mathematics from this point and expand to cover the spatial averaging later. We can solve Eq. (2) in terms of the distribution function of the 
$Z+1$ charged ion. Using integration by parts, one can show that

$$
\vec{v}_{a p p}^{l o c a l}=\frac{\int d^{3} v\left[\vec{v}+\frac{\omega_{c} \tau}{B}(\vec{v} \times \vec{B})+\frac{\omega_{c}^{2} \tau^{2}}{B^{2}}(\vec{v} \cdot \vec{B}) \vec{B}\right] \frac{n_{b} Q\left(\left|\vec{v}-\vec{b}_{b}\right|\right)}{1+\omega_{c}^{2} \tau^{2}} f_{n+1}(\vec{v}-\vec{V})}{\int d^{3} v n_{b} Q\left(\left|\vec{v}-\vec{v}_{b}\right|\right) f_{n+1}(\vec{v}-\vec{V})} .
$$

Inspection of Eq. (3) reveals that the apparent velocity is dependent on only one velocity, the apparent velocity of the $Z_{n}$ charged ion immediately after charge exchange

$$
\vec{v}_{n}=\frac{\int d^{3} v \vec{v} n_{b} Q\left(\left|\vec{v}-\vec{v}_{b}\right|\right) f_{n+1}(\vec{v}-\vec{V})}{\int d^{3} v n_{b} Q\left(\left|\vec{v}-\vec{v}_{b}\right|\right) f_{n+1}(\vec{v}-\vec{V})} .
$$

Equation (3) contains an operator that acts upon this velocity. In particular, this operator describes how the velocity vector is twisted around the magnetic field by the ion gyro motion during the lifetime $\tau$. One can reduce the three dimensional velocity integrals into one dimensional integrals under the assumption of a Maxwellian distribution of $Z_{n+1}$ charged ions. The result is given by

$$
\begin{aligned}
\vec{v}_{n} & =\vec{V}+\frac{\left(\vec{v}_{b}-\vec{V}\right)}{\left|\vec{v}_{b}-\vec{V}\right|} \frac{\int_{-\infty}^{\infty} d \nu n_{b} Q\left(\left|v_{t h} \nu+\right| \vec{v}_{b}+\vec{V}||\right) \frac{n v_{t h}}{2 \sqrt{\pi}}\left[1+\frac{v_{t h}}{\left|\overrightarrow{v_{b}}-\vec{V}\right|} \nu\right]\left[\frac{v_{t h}}{\left|\overrightarrow{v_{b}}-\vec{V}\right|}-2 \nu\right] e^{-\nu^{2}}}{\int_{-\infty}^{\infty} d \nu n_{b} Q\left(\left|v_{t h} \nu+\right| \vec{v}_{b}+\vec{V}||\right) \frac{n}{\sqrt{\pi}}\left[1+\frac{v_{t h}}{\left|\vec{v}_{b}-\vec{V}\right|} \nu\right] e^{-\nu^{2}}} \\
& =\vec{V}+\alpha \frac{\left(\vec{v}_{b}-\vec{V}\right)}{\left|\vec{v}_{b}-\vec{V}\right|} .
\end{aligned}
$$

Hence, the velocity immediately after charge exchange of the $Z_{n}$ charged ion is the true velocity of the $Z_{n+1}$ charged impurity ion that we are interested in, plus a correction due to the energy-dependent cross-section correction, which is directed along the unit collision vector between the beam neutral and ion. Equation (5) must be inserted into the operator shown in Eq. (3) to give the final result for the apparent velocity

$$
\vec{v}_{a p p}^{\text {local }}\left(\vec{v}_{n}\right)=\frac{1}{1+\omega_{c}^{2} \tau^{2}}\left[\vec{v}_{n}+\frac{\omega_{c} \tau}{B}\left(\vec{v}_{n} \times \vec{B}\right)+\frac{\omega_{c}^{2} \tau^{2}}{B^{2}}\left(\vec{v}_{n} \cdot \vec{B}\right) \vec{B}\right] .
$$

As discussed earlier, this result is only valid for a local point measurement, which is not the case for a typical CER chord. The consequence of this is that rather than the local integral described by Eq. (2), one must compute the line-average distribution function

$$
\vec{v}_{a p p}=\frac{\int d^{3} v \int d l \vec{v} f_{n}}{\int d^{3} v \int d l f_{n}}
$$

where the $\int d l$ represents the spatial averaging that occurs as a result of the CER being a LOS measurement. In the case that the ion gyro orbit is much less than the gradient scale 
length $\left(\rho_{i} \ll L_{p}\right)$, then the velocity and line integrals can be reversed. With the spatial averaging, some of the convenient cancellations that were made in the local derivation are no longer possible, however a structurally similar result is still obtained. We must replace $v_{n}$ in Eq. (6) with a slightly modified velocity

$$
\begin{aligned}
\vec{v}_{n}^{*}= & \vec{V}\left\{\int_{-\infty}^{\infty} d \nu Q\left(\left|v_{t h} \nu+\right| \vec{v}_{b}+\vec{V}||\right)\left[1+\frac{v_{t h}}{\left|\vec{v}_{b}-\vec{V}\right|} \nu\right] e^{-\nu^{2}}\right\}+ \\
& \frac{\left(\vec{v}_{b}-\vec{V}\right)}{\left|\vec{v}_{b}-\vec{V}\right|}\left\{\int_{-\infty}^{\infty} d \nu Q\left(\left|v_{t h} \nu+\right| \vec{v}_{b}+\vec{V}||\right) \frac{v_{t h}}{2}\left[1+\frac{v_{t h}}{\left|\vec{v}_{b}-\vec{V}\right|} \nu\right]\left[\frac{v_{t h}}{\left|\vec{v}_{b}-\vec{V}\right|}-2 \nu\right] e^{-\nu^{2}}\right\} .
\end{aligned}
$$

Then, the measured, line-of-sight velocity is given by

$$
v_{\text {meas }}=\frac{\int d l \hat{s} \cdot n_{b} n \vec{v}_{a p p}^{l o c a l}\left(\vec{v}_{n}^{*}\right)}{\int d l n_{b} n \int_{-\infty}^{\infty} d \nu Q\left(\left|v_{t h} \nu+\right| \vec{v}_{b}+\vec{V}||\right)\left[1+\frac{v_{t h}}{\left|\vec{v}_{b}-\vec{V}\right|} \nu\right] e^{-\nu^{2}}},
$$

where $\hat{s}$ is the unit vector of the viewing chord.

\section{SEPARATION OF CORRECTIONS FROM ROTATION}

We seek a set of views that will allow us to uniquely separate out the atomic physics corrections from the true rotation velocities, without relying on the results from detailed atomic physics modeling. A plasma cross-section is shown in Fig. 2, and we consider making a measurement of the rotation velocity on the solid-lined flux surface. In this example, the positive toroidal direction is into the page, and the positive poloidal direction is clock-

wise around the flux surface. For simplicity of the explanation only, we consider the CER measurements to be local point-measurements, and that both the poloidal and toroidal velocities are flux surface quantities. Superimposed on Fig. 2 are five CER views, all on the same flux surface, directed tangentially, "radially" and vertically. Considering only the dominant corrections from Eq. (6), the tangential and radial views will be subject to the standard energy-dependent cross-section correction, whilst the vertical views will need to account for the gyro-orbit cross-section correction. Specifically, the tangential measurement will contain the toroidal velocity plus a correction, $V_{T}=V_{\phi}+V^{\prime}$. The two "radial" measurements are made at the top and bottom of the flux surface. Although these views are radial from the machine-sense, on the flux surface of interest they are sampling the poloidal 
velocity. The measurements will again be subject to the energy-dependent cross-section, $V_{R}^{ \pm}= \pm V_{\theta}+V^{\prime}$, remembering that the poloidal velocity is oppositely directed on the two sides of the flux surface. In an ideal world, these two measurements alone would be sufficient for poloidal velocity measurements - one would merely have to add and subtract the two views to determine the correction and poloidal velocity respectively. However, it turns out that the poloidal velocity is an order of magnitude smaller than the energy-dependent cross-section correction, and so while radial views are good for measuring this correction, they are not well-suited to measuring poloidal velocities. In addition then, a vertical view on this flux surface is also used to measure the poloidal velocity, but this time, rather than being affected by the standard energy-dependent cross-section correction as was the case for the radial views, this view must account for a gyro-orbit correction, $V_{V}=-V_{\theta}-\omega_{c} \tau V^{\prime}$. At $B \sim 2 \mathrm{~T}$, the value of $\omega_{c} \tau$ is less than 0.1 for the $C^{6+} \rightarrow C^{5+}(n=8 \rightarrow 7)$ transition (where $\tau \sim 1 \mathrm{~ns}$ ), and therefore the correction and the poloidal velocity are of the same magnitude. The final view to consider is one that nominally passes through the center, and for this geometry it measures the true radial velocity (which is assumed to be zero), plus a gyro-orbit correction, $V_{V}^{0}=\omega_{c} \tau\left(V_{T} B_{\theta}-V_{R}^{+} B_{\phi}\right) / B$. So this view is a linear combination of two of the other views, multiplied by the effective lifetime $\tau$. Hence, with this set of tangential, radial, and vertical views, the atomic physics quantities can be backed out from the measurement, leaving the true rotation velocity. In the following section, we present modeling that aids in devising a more complete set of views to measure the rotation profiles.

\section{MODELING}

The rotation profiles are generated in terms of the neoclassical flux-surface quantities $k(\rho)$ and $\Omega(\rho)$

$$
\vec{V}=k \vec{B}+R \Omega \hat{\phi}
$$

where $R$ is the major radius, and $\hat{\phi}$ is the unit toroidal vector. Resolving into poloidal and toroidal components

$$
\begin{aligned}
V_{\theta} & =k B_{\theta}, \\
V_{\phi} & =k B_{\phi}+R \Omega .
\end{aligned}
$$

We use $k$ and $\Omega$ profiles from a real DIII-D discharge to model the rotation profiles. In 
combination with the other relevant plasma profiles, we then compute the measured LOS velocities using Eq. (9). This represents the simulated CER measurement. In practice, we perform the $\int d l$ integral in Eq. (9) by dividing the chord into 11 regions throughout the neutral beam. Increasing the number of segments does not alter the numerical approximation. We then attempt to recover the inputted $k$ and $\Omega$ profiles based on the simulated LOS velocities (with appropriate noise levels added). If we can successfully recover these profiles, then our system is capable of self-consistently separating out the true rotation from the atomic physics effects. In performing the fits, we model the various profiles with cubic splines, and hence the fit involve a non-linear least squares fit for the knot locations and knot values of $k$ and $\Omega$. Simultaneously, we try to determine the value for the effective lifetime $\tau$, and the energy-dependent cross-section function $Q$. Specifically, we fit for the coefficients $p$ and $q$ in the von Hellerman et al. [3] formulation of the energy-dependent cross-section

$$
Q=Q_{0} \frac{X^{p}}{1+X^{q}}
$$

where $X=v / v_{m}$, and $v_{m}$ is approximately the velocity with maximum emission rate.

Figure 3 shows the set of CER views used in the modeling analysis. The dotted view lines represent chords that existed during the 2003 experimental campaign, which had 24 tangential, 17 vertical, and 2 radial sight lines. Modeling performed with this set of views revealed that the recovered rotation profiles suffered from more than a factor of two greater error than was input into the simulation. This led to the search for improved views to reduce this uncertainty.

Following the untangling procedure outlined in Sec. III, we identified certain aspects in the 2003 design that needed improvement if we were to separate the atomic physics corrections from the rotation. The first affects the determination of $\tau$, which conceptually requires a view through the plasma center. In the non-local treatment, vertical views close to the plasma center (or, equivalently, views that are predominantly radial from the plasma-sense) are equally useful. In 2003, there was only 2 vertical chords inside of $\rho \sim 0.3$, so clearly the upgrade called for new vertical chords close to the magnetic axis. Additional vertical chords are also desirable inside of $\rho \leq 0.5$ simply because this is where the gyro-orbit correction is most important. The other desirable modification involves the radial chords. With only two present in 2003, the determination of $p$ and $q$ was not really possible. One could conceive of an array of radial chords similar to the tangential and vertical arrays. However, from 
a plasma physics standpoint, this is not a great utilization of hardware, since these radial chords mainly measure cross-section corrections, rather than the rotation with which we are fundamentally concerned. Fortunately, our modeling shows that the addition of just two new radial type chords are sufficient to accurately pin down $p$ and $q$.

The solid view lines in Fig. 3 show the additional chords ( 8 new vertical chords, and 2 new radial chords) that were decided upon through modeling, allowing for greatly improved extraction of the poloidal velocity. These views have been installed on DIII-D for use in the 2004 experimental campaign. With this full set of views, the rotation profiles can be recovered to the same level as the input noise.

A typical reconstruction of the rotation profiles from the simulated measured velocities based on the 2004 sight lines is shown in Fig. 4. The dotted curves represent the input model splines. The reconstructed profiles are shown with solid curves, with the error bands shown as dashed. The errors are determined through Monte-Carlo simulation, whereby the simulated velocities are perturbed by amounts consistent with their error bars. The modeled effective lifetime $\tau$ is allowed to vary throughout the plasma. This is because, even though the lifetime of a particular state is fixed by atomic physics, the effective lifetime, considering cascading from different energy levels, is a function of plasma parameters including density. Nonetheless, when trying to solve for $\tau$, we do not attempt to recover the profile, but rather just a single value. As we move from the plasma center to the edge, the ion temperature is decreasing. Hence, the gyro-orbit correction becomes less important. In fact, the value of $\tau$ at the edge does not really influence the measured velocities, and so there is little hope (or point) in trying to fit it.

The recovered profiles are in excellent agreement with the input profiles, and the error bands for the poloidal and toroidal velocities are consistent with the input error on the simulated velocities. Also note that we do a good job in empirically measuring the energydependent cross-section as indicated by the tight error bands around Fig. 4(f).

\section{RESULTS}

The analysis of a quiescent H-mode (QH-mode) discharge is presented in Fig. 5. This particular discharge, as is common for QH-mode, had relatively high ion temperature $T_{i}(0) \sim 15 \mathrm{keV}$, and moderate line average density $\bar{n}_{e} \sim 2 \times 10^{19} \mathrm{~m}^{-3}$. Due to the high ion 
temperature, we can expect substantial atomic physics corrections to be necessary for the CER measurements. Figures 5(a-b) show the CER measured LOS velocities for the vertical and tangential viewing arrays. The solid curves show the reprojected LOS velocities based on the fitted toroidal and poloidal velocities. The reprojections are in very good agreement with the measurements, and are significantly improved compared with a fit that does not include either atomic physics corrections. The spline fits for the corrected toroidal and poloidal velocity are shown in Figs. 5(c-d). Overplotted with a dashed curve is the fitted profile that results from an analysis that does not include any atomic physics corrections. Note that the correction to the toroidal velocity due to the energy-dependent cross-section correction is more than $100 \mathrm{~km} / \mathrm{s}$ at the magnetic axis where the rotation is maximum. The effect on the poloidal velocity is somewhat less pronounced in absolute magnitude, although it still amounts to a $25 \%$ correction. The gyro-orbit correction appears to be smaller here due to a couple of competing effects. The vertical views on DIII-D are not ideally vertical, and some indeed have substantial radial components (and even a little toroidal). So the vertical views are subject to some modification from the ordinary energy-dependent cross-section correction and this happens to counter the gyro-orbit correction. In addition, the spatial averaging of the chord through the vertically elongated beam tends to further weaken the gyro-orbit correction. The effective lifetime is determined in this discharge to be $\tau=1.70 \pm 0.15$ ns. This is somewhat larger than found in the modeling performed by Bell and Synakowski [2]. However, a more systematic study of the effective lifetime in a variety of plasma conditions is required before any conclusions should be made. Also, the time-slice to time-slice variability of $\tau$ appears to be larger than the error estimated from the non-linear fit analysis, indicating that further refinements in the analysis are still required.

There are several improvements that are needed before the work can be considered complete. At present, the current analysis only considers the full energy component of the beam neutrals. Furthermore, a tiny fraction $(\sim 0.1 \%)$ of beam neutrals in the $n=2$ state can significantly alter the curve of the energy-dependent cross-section shown in Fig. 1. Nonetheless, we have some strong indication that the model form of the energy-dependent cross-section that we use in Eq. (13) is a good approximation. Two of the CER tangential view chords on DIII-D look at the exact same location, but with slightly differing toroidal angles [Fig. 6(a)]. In fact, the difference is only about 5\%. Even so, if we look at the raw LOS measured velocities from these two chords, we see in Fig. 6(b) that they differ by an amount much 
greater than can be explained by the difference in their viewing geometry. The discrepancy, of course, is due to the energy-dependent cross-section correction. When we apply this correction, using the form from Eq. (13), as well as the viewing geometry correction, we see that the results are in near perfect agreement.

\section{Acknowledgments}

This work is supported by the U. S. Department of Energy under DE-AC02-76CH03073, DE-FC02-04ER54698, and DE-AC05-000R22725.

[1] R. Isler, Phys. Rev. Lett. 38, 1359 (1977).

[2] R. E. Bell and E. J. Synakowski, AIP Conference Proceedings 547, 39 (2000).

[3] M. von Hellerman, P. Breger, J. Frieling, R. König, W. Mandl, A. Maas, and H. P. Summers, Plasma Phys. Control. Fusion 37, 71 (1995). 


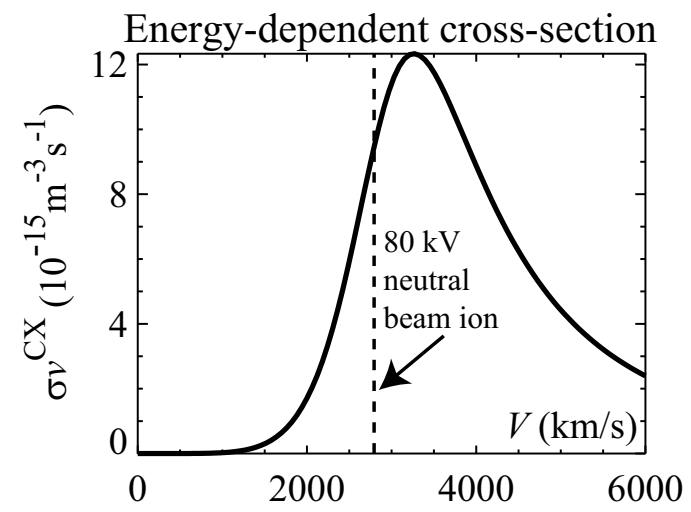

FIG. 1: Charge exchange cross-sectional area as a function of velocity for the $D^{0}+C^{6+} \rightarrow D^{+}+$ $C^{5+}(n=8 \rightarrow 7)$ reaction. In DIII-D, the beam energies are such that we operate on the left hand side of the maximum.

W. M. Solomon 


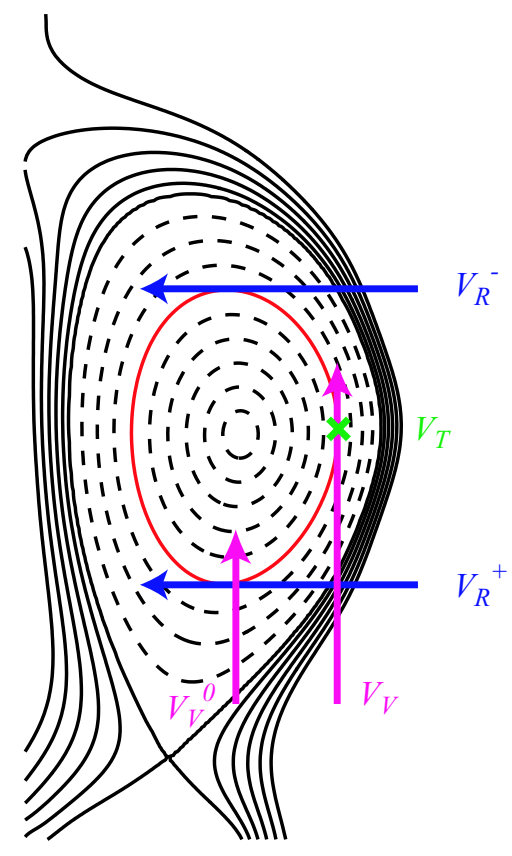

FIG. 2: Conceptual view chords required to unravel the atomic physics corrections from the plasma rotation.

W. M. Solomon 


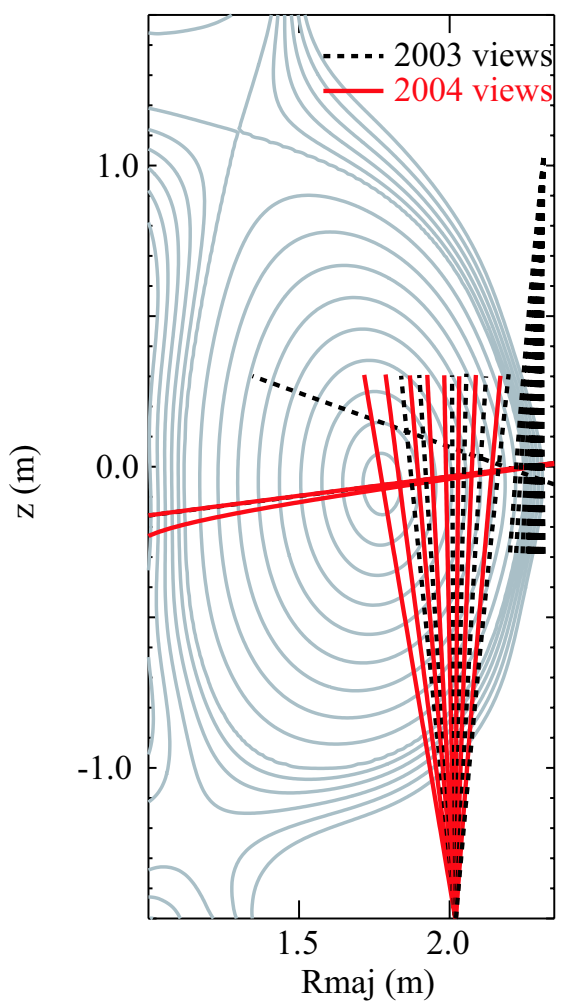

FIG. 3: CER viewing geometry on DIII-D. Shown are the chords present in the 2003 experimental campaign (dashed) and the new chords for 2004 (8 verticals and 2 radials; solid).

W. M. Solomon 

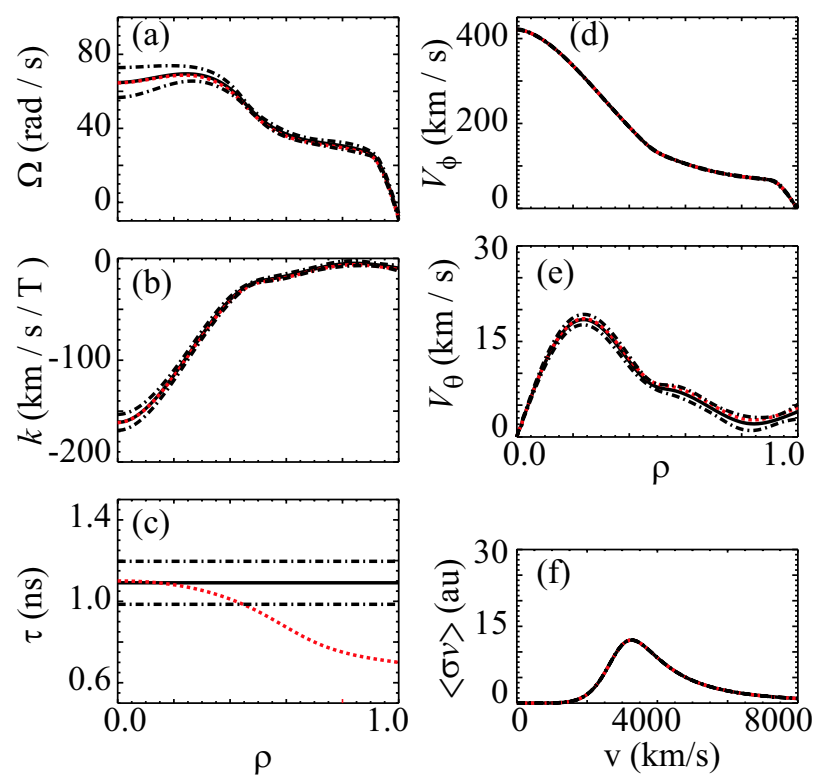

FIG. 4: Simulated (dotted) and reconstructed (solid) profiles for (a) $\Omega$, (b) $k$, (c) effective lifetime $\tau$, (d) toroidal velocity $V_{\phi}$, (e) poloidal velocity $V_{\theta}$, and (f) the energy-dependent cross-section $\langle\sigma v\rangle$ using the full set of CER view chords available in 2004. The error bands for the reconstructions are shown by the dashed lines.

W. M. Solomon 

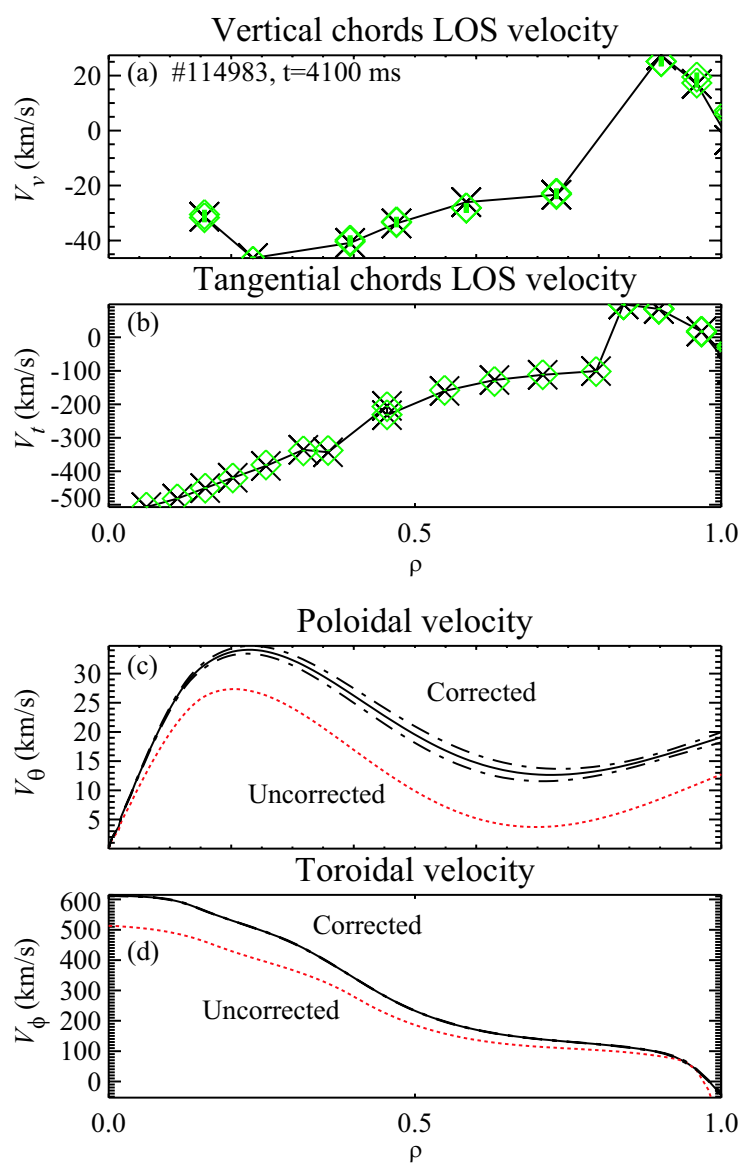

FIG. 5: LOS velocities as measured by the (a) vertical and (b) tangential viewing arrays during a QH-mode discharge. Raw measurements are shown with diamonds. The error bars, although shown, are smaller than the symbol size. The reconstructed LOS velocities are depicted with crosses and joined with a solid line. Profiles (along the outer midplane) for (c) the poloidal velocity, and (d) the toroidal velocity. The solid curves represent the fits to the measured data (error bands are shown with a dashed curve), whilst the dotted curve is the fit that would have been obtained had all the atomic physics corrections been ignored.

\section{W. M. Solomon}



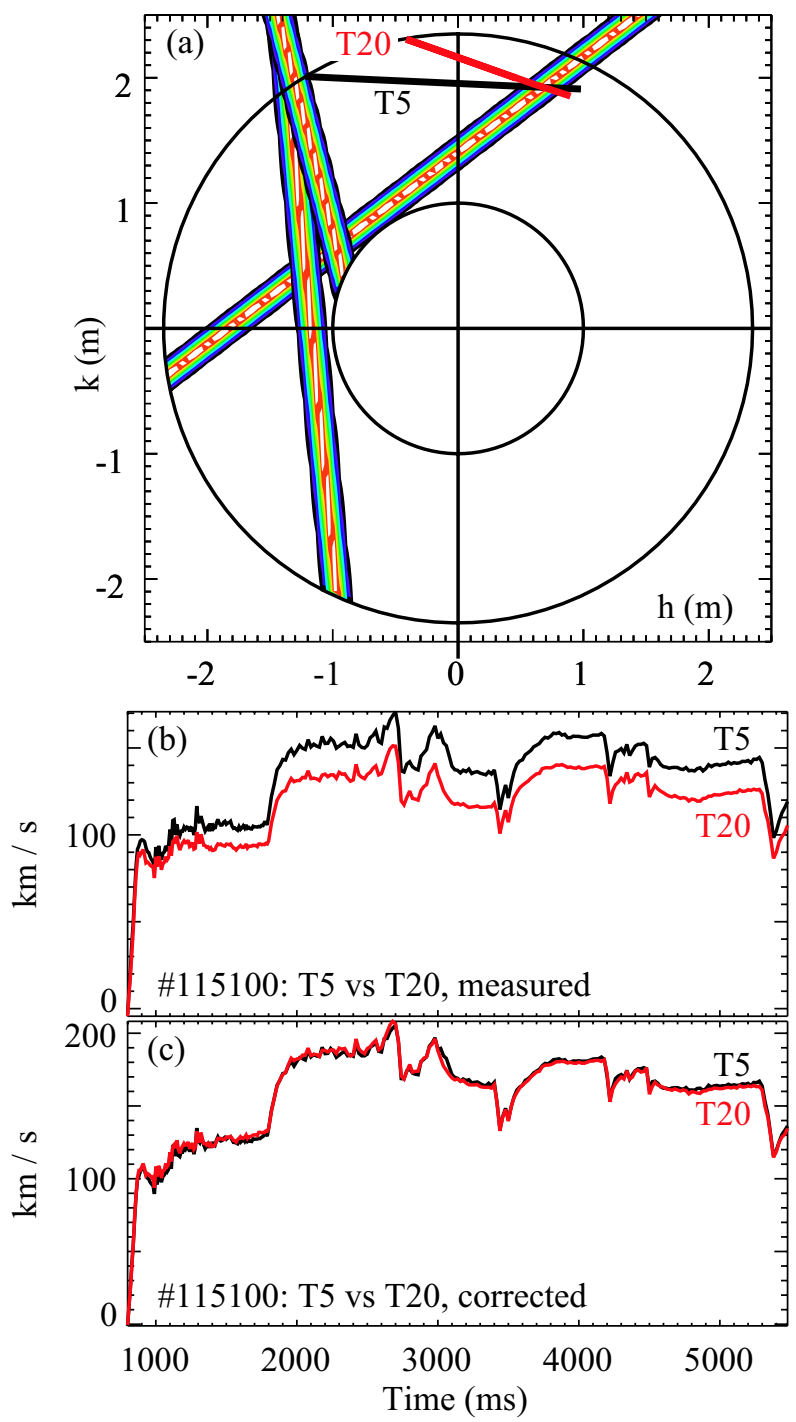

FIG. 6: (a) Plan view of DIII-D showing the neutral beams viewed by the various CER chords, and specifically chords labeled "T5" and "T20", which look at the exact same location in the plasma, but with slightly differing viewing geometries $(\sim 5 \%)$. The positive $k$-axis is in the direction of 0 degrees and the $h$-axis is at 90 degrees. The raw, uncorrected measurements for these two chords (b) show a large discrepancy, which cannot be explained by the viewing geometry effect alone. When the energy-dependent cross-section correction and geometry effects are taken into consideration, near perfect agreement is obtained between the two channels (c). 


\section{External Distribution}

Plasma Research Laboratory, Australian National University, Australia

Professor I.R. Jones, Flinders University, Australia

Professor João Canalle, Instituto de Fisica DEQ/IF - UERJ, Brazil

Mr. Gerson O. Ludwig, Instituto Nacional de Pesquisas, Brazil

Dr. P.H. Sakanaka, Instituto Fisica, Brazil

The Librarian, Culham Laboratory, England

Mrs. S.A. Hutchinson, JET Library, England

Professor M.N. Bussac, Ecole Polytechnique, France

Librarian, Max-Planck-Institut für Plasmaphysik, Germany

Jolan Moldvai, Reports Library, Hungarian Academy of Sciences, Central Research Institute for Physics, Hungary

Dr. P. Kaw, Institute for Plasma Research, India

Ms. P.J. Pathak, Librarian, Institute for Plasma Research, India

Ms. Clelia De Palo, Associazione EURATOM-ENEA, Italy

Dr. G. Grosso, Instituto di Fisica del Plasma, Italy

Librarian, Naka Fusion Research Establishment, JAERI, Japan

Library, Laboratory for Complex Energy Processes, Institute for Advanced Study, Kyoto University, Japan

Research Information Center, National Institute for Fusion Science, Japan

Dr. O. Mitarai, Kyushu Tokai University, Japan

Dr. Jiangang Li, Institute of Plasma Physics, Chinese Academy of Sciences, People's Republic of China

Professor Yuping Huo, School of Physical Science and Technology, People's Republic of China

Library, Academia Sinica, Institute of Plasma Physics, People's Republic of China

Librarian, Institute of Physics, Chinese Academy of Sciences, People's Republic of China

Dr. S. Mirnov, TRINITI, Troitsk, Russian Federation, Russia

Dr. V.S. Strelkov, Kurchatov Institute, Russian Federation, Russia

Professor Peter Lukac, Katedra Fyziky Plazmy MFF UK, Mlynska dolina F-2, Komenskeho Univerzita, SK-842 15 Bratislava, Slovakia

Dr. G.S. Lee, Korea Basic Science Institute, South Korea

Institute for Plasma Research, University of Maryland, USA

Librarian, Fusion Energy Division, Oak Ridge National Laboratory, USA

Librarian, Institute of Fusion Studies, University of Texas, USA

Librarian, Magnetic Fusion Program, Lawrence Livermore National Laboratory, USA

Library, General Atomics, USA

Plasma Physics Group, Fusion Energy Research Program, University of California at San Diego, USA

Plasma Physics Library, Columbia University, USA

Alkesh Punjabi, Center for Fusion Research and Training, Hampton University, USA

Dr. W.M. Stacey, Fusion Research Center, Georgia Institute of Technology, USA

Dr. John Willis, U.S. Department of Energy, Office of Fusion Energy Sciences, USA

Mr. Paul H. Wright, Indianapolis, Indiana, USA 
The Princeton Plasma Physics Laboratory is operated by Princeton University under contract with the U.S. Department of Energy.

\author{
Information Services \\ Princeton Plasma Physics Laboratory \\ P.O. Box 451 \\ Princeton, NJ 08543
}

Phone: 609-243-2750

Fax: 609-243-2751

e-mail: pppl_info@pppl.gov

Internet Address: http://www.pppl.gov 